\title{
A discretization result for some optimization problems in framework spaces with polyhedral obstacles and the Manhattan metric
}

\author{
Justo Puerto ${ }^{1,2,3}$ \\ Department of Statistic and OR \\ Institute of Mathematics University of Seville (IMUS) \\ Universidad de Sevilla \\ Sevilla, Spain \\ Moisés Rodríguez-Madrena ${ }^{1,2,3}$ \\ Department of Statistic and OR \\ Institute of Mathematics University of Seville (IMUS) \\ Universidad de Sevilla \\ Sevilla, Spain
}

\begin{abstract}
In this work we consider the shortest path problem and the single facility Weber location problem in any real space of finite dimension where there exist different types of polyhedral obstacles or forbidden regions. These regions are polyhedral sets and the metric considered in the space is the Manhattan metric. We present a result that reduce these continuous problems into problems in a "add hoc" graph, where the original problems can be solved using elementary techniques of Graph Theory. We show that, fixed the dimension of the space, both the reduction and the resolution can be done in polynomial time.
\end{abstract}

Keywords: Metric optimization problems with obstacles. Network optimization. Finite dominating sets. 


\section{Introduction}

The Manhattan metric $\ell_{1}$ is a well-known distance measure very useful in many realistic applications: wire layout, circuit design, plant and facility layout, urban transportation and robot motion, among others.

Associated to the Manhattan metric $\ell_{1}$ we find the rectilinear paths. A rectilinear path $R P_{X, Y}$ between two point $X, Y \in \mathbb{R}^{n}$ is a path from $X$ to $Y$ formed by the union of a finite set of rectilinear segments parallel to the reference axes. Indeed, if we denote by $\mathcal{R} \mathcal{P}_{X, Y}$ the set of rectilinear paths from $X$ to $Y$, then

$$
\ell_{1}(X, Y)=\min \left\{\operatorname{length}\left(R P_{X, Y}\right): R P_{X, Y} \in \mathcal{R} \mathcal{P}_{X, Y}\right\}
$$

where length $\left(R P_{X, Y}\right)$ is the sum of the lengths of the segments defining $R P_{X, Y}$. We restrict ourselves in what follows to consider the metric $d=\ell_{1}$.

In real world applications, it is very often needed to consider constraints which change the properties of the distance measures in the framework space. These regions model traveling limitations as, for example, protected or military areas, mountain ranges, lakes, or, on small scale, machinery in an industrial plant.

These constraint regions can be:

- Weighted obstacles: traveling through the region is allowed but it increases a proportional cost on the traveled distance [6].

- Barriers: traveling through the region is forbidden $[2,3,5]$.

In addition, in facility location problems we can consider:

- Forbidden regions: traveling through the region is allowed but locating a facility is not allowed [1].

- Congested regions: placing a facility is prohibited but traveling through the region is allowed, although it carries on a proportional cost on the traveled distance.

1 This research has been partially supported by Spanish Ministry of Economia and Competitividad/FEDER grants number MTM2016-74983-C02-01.

2 Email: puerto@us.es

3 Email: madrena@us.es 
Clearly, in facility location applications locating a facility in a barrier is forbidden since it has no sense.

We are interested in rectilinear constraint regions which are regions shaped like linear polyhedral sets. Linear polyhedral sets are sets delimited by a connected union of a finite set of isotetic parallepipeds (parallepideds whose edges of dimension one are parallel to the reference axes). This is the framework in which we consider the problems.

\section{The problems}

In $\mathbb{R}^{n}$ with $n \geq 2$, let $B_{1}, \ldots, B_{m}$ be a set of rectilinear constraint regions pairwise disjoint, where each one of them can be of a different type from one another. The restriction set $\mathcal{B}=\bigcup_{i=1}^{m} B_{i}$ induced a metric $d_{\mathcal{B}}$ in $\mathbb{R}^{n}$ called the rectilinear restriction region metric. Given two points $X, Y \in \mathbb{R}^{n}$, the metric $d_{\mathcal{B}}(X, Y)$ is the minimum length for a rectlinear path between $X$ and $Y$ taking into account how the different types of barriers in $\mathcal{B}$ change the value of the Manhattan metric.

In this context, we want to solve the shortest path problem and the minisum (Weber) single facility location problem $[7,8]$. The first one consists on finding the $d_{\mathcal{B}}$-minimum rectangular path between two points $X, Y \in \mathbb{R}^{n}$. The second problem consists on, given a set of existing facilities $\left\{E x_{1}, \ldots, E x_{K}\right\} \subseteq$ $\mathbb{R}^{n}$, finding the location $X \in \mathbb{R}^{n}$ of a new facility solving the problem:

$$
\begin{aligned}
& \min \sum_{k=1}^{K} w_{k} d_{\mathcal{B}}\left(X, E x_{k}\right) \\
& \text { s.t: } X \in \mathbb{R}^{n},
\end{aligned}
$$

where $w_{k}>0$ is the weight for facility $E x_{k}$, for each $k \in\{1, \ldots, K\}$.

\section{The rectilinear construction grid}

Let us denote by $\mathcal{P}(\mathcal{B})$ the set of extreme points of $\mathcal{B}$, and let $\mathcal{E} x$ be: the set $\{X, Y\}$ of the two points which we want to join by a $d_{\mathcal{B}}$-minimum rectangular path, if we are considering the shortest path problem; the set $\left\{E x_{1}, \ldots, E x_{K}\right\}$ of existing facilities, if we are considering the single facility location problem. Algorithm 1 builds a graph $\mathcal{N}_{\ell_{1}}$ called the base rectilinear construction grid of the problem, which is the basis of our discretization result. 


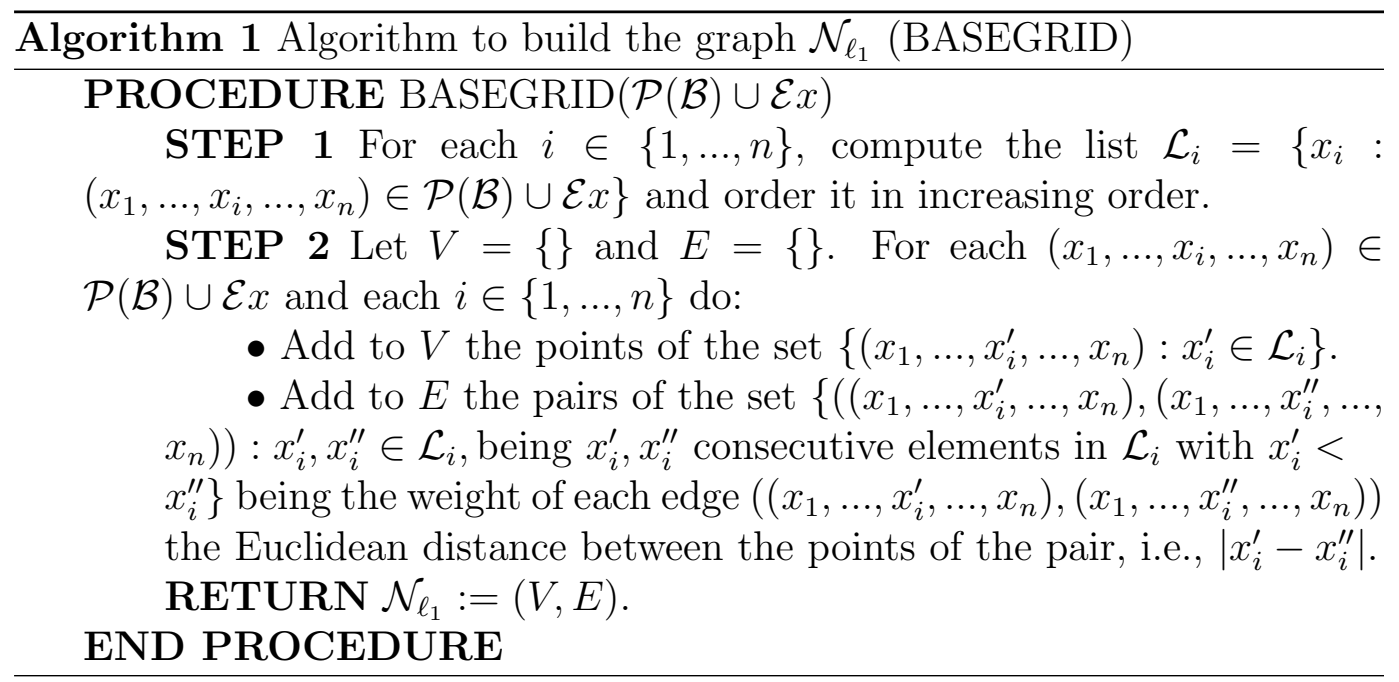

Note that, fixed the dimension $n$ of the space, Algorithm 1 is polynomial. Obtained the base rectilinear construction grid, we make to $\mathcal{N}_{\ell_{1}}$ a polynomial transformation (removing some vertices, changing the weight of the edges, etc.) in order to obtain the rectilinear construction grid $\mathcal{N}_{d_{\mathcal{B}}}$. The transformation above depends on the constraint regions, and their type, present in the framework space. The grid $\mathcal{N}_{d_{\mathcal{B}}}$ is an extension, to higher dimension and more general constraint regions, of the one that appears in [4].

\section{Main results}

We show how the shortest path problem reduces to finding another shortest path in the resulting graph $\mathcal{N}_{d_{\mathcal{B}}}$ and can be solved by using any of the wellknown algorithms for this problem, for instance Dijsktra's algorithm. Concerning the minisum (Weber) single facility location problem we prove that there exists a finite dominating set defined by the set of vertices of $\mathcal{N}_{\mathcal{d}_{\mathcal{B}}}$. Finally, we show that, fixed the dimension of the space, both the reduction and the resolution of these continuous problems can be done in polynomial time.

\section{References}

[1] Butt, S. E., \& Cavalier, T. M. (1996). An efficient algorithm for facility location in the presence of forbidden regions. European Journal of Operational Research, 90(1), 56-70.

[2] de Berg, M., van Kreveld, M., Nilsson, B. J., \& Overmars, M. H. (1990, July). Finding shortest paths in the presence of orthogonal obstacles using a combined 
$L_{1}$ and link metric. In Scandinavian Workshop on Algorithm Theory (pp. 213224). Springer, Berlin, Heidelberg.

[3] De Rezende, P. J., Lee, D. T., \& Wu, Y. F. (1985, June). Rectilinear shortest paths with rectangular barriers. In Proceedings of the first annual symposium on Computational geometry (pp. 204-213). ACM.

[4] Klamroth, K. (2006). "Single-facility location problems with barriers." Springer Science \& Business Media.

[5] Larson, R. C., \& Sadiq, G. (1983). Facility locations with the Manhattan metric in the presence of barriers to travel. Operations Research, 31(4), 652-669.

[6] Lee, D. T., Yang, C. D., \& Chen, T. H. (1991). Shortest rectilinear paths among weighted obstacles. International Journal of Computational Geometry \& Applications, 1(02), 109-124.

[7] Nickel, S., Puerto, J., (2005) Location Theory: A unified approach. Springer Series in Operations Research and Decision Theory. ISBN: 3-540-24321-6.

[8] Puerto, J., Rodriguez-Chia, A.M., (2011). On the structure of the solution set for the single facility location problem with average distances. Mathematical Programming 128:373-401. 